\title{
PROPOSTA DE EXTENSÃO DO PROCESS CLASSIFICATION FRAMEWORK DA APQC PARA OBTENÇÃO DE UMA REFERÊNCIA PARA OS PROCESSOS DE OPERAÇÃO DE PSS
}

Carolina Queiroz (queirozc@usp.br) - Departamento de Engenharia de Produção, Escola de Engenharia de São Carlos, Universidade de São Paulo

Marina de Pádua Pieroni (mdpp@mek.dtu.dk) - Technical University of Denmark (DTU), Department of Mechanical Engineering

Caio Augusto Nunes Marques (caio.nunes.marques@gmail.com) - Departamento de Engenharia de Produção, Escola de Engenharia de São Carlos, Universidade de São Paulo

Sânia da Costa Fernandes (saniafernandes@usp.br) - Departamento de Engenharia de Produção, Escola de Engenharia de São Carlos, Universidade São Paulo

Henrique Rozenfeld (roz@usp.br) - Departamento de Engenharia de Produção, Escola de Engenharia de São Carlos, Universidade São Paulo

\section{RESUMO}

Durante o desenvolvimento de um sistema produto-serviço (PSS - Product-Service System) deve-se definir, além de outros elementos, a infraestrutura que garanta a sua operação. O foco deste trabalho está nos processos de middle of life (MOL) do ciclo de vida de PSS, que são os processos de venda, uso, manutenção e fornecimento de serviços. Para apoiar a especificação desses processos, uma boa prática é o uso de modelos de referência que contenham esses processos de operação. Nesse contexto, o trabalho objetiva a configuração dos processos de operação de PSS na estrutura de um modelo de referência de processos de negócio, o Process Classification Framework da APQC, e com isso iniciar a proposição de um modelo de referência para PSS constituído de uma extensão do PCF. A metodologia adotada abrange uma combinação de revisão bibliográfica no contexto de servitização, modelos de referência de processos de negócio e modelos de design de PSS e análise por similaridade de processos e atividades de PSS para a extensão do modelo. O resultado é uma tentativa de se elaborar um modelo de referência orientado aos processos de operação de um PSS a partir da extensão de um modelo de referência já existente.

Palavras-chave: PSS; modelo de referência; operação; middle of life

Área: Desenvolvimento de Sistemas Produto-Serviço (PSS)

\section{INTRODUÇÃO}

Sistema produto-serviço (PSS - Product-Service System) é a combinação de produtos e serviços de forma integrada com o objetivo de aumentar o valor percebido pelos clientes e garantir menor impacto ambiental (BAINES et al., 2007). Para fornecer PSS, as empresas devem migrar seu modelo de negócio da venda de produtos físicos para oferta da combinação integrada de produtos e serviços (BOEHM; THOMAS, 2013, p.252). Essa mudança na oferta é definida por Baines et al., (2009, p 555) como servitização.

Este trabalho permeia o contexto da "metodologia para servitização" que está sendo desenvolvida no grupo de pesquisa ao qual os autores deste trabalho participam. A metodologia possui o objetivo de auxiliar as empresas de manufatura para obterem êxito no 
processo de servitização. Para isto, um framework foi elaborado e é utilizado para apoiar a transição do modelo de negócio baseado na oferta de produtos para um modelo de negócio de PSS. Tal framework, denominado PSS Transition Framework, é composto por nove fases iterativas, nas quais são aplicados métodos que objetivam a entrega de um modelo de negócios de PSS completo (PIERONI et al., 2016).

No desenvolvimento do PSS a partir do PSS Transition Framework (PIERONI et al., 2016), o novo modelo de negócio é especificado a partir da análise do negócio, seguida pela proposição de valor - fase na qual há o entendimento dos clientes e stakeholders, de modo que ocorra a identificação de suas necessidades e dos problemas que podem ser resolvidos com a proposta de valor. A partir disto, o modelo de negócio atual e o business case são definidos, e, então, realiza-se a definição dos processos de operação do PSS. Essa definição é obtida por meio da definição da arquitetura dos processos de negócio, seu detalhamento, preparação da cadeia de valor e lançamento da cadeia de valor.

Na literatura, há inúmeros modelos de referência de processos de negócio. No entanto, há uma escassez de modelos para a operação de PSS. Esse assunto tem se tornado presente nos campos de pesquisa para a aplicação de modelos de desenvolvimento em PSS por meio da adaptação de modelos de referência conhecidos ou pela combinação de modelos orientados à manufatura e serviços (CASTAGNARI, 2016). Um dos modelos mais utilizados é o Process Classification Framework (PCF) da APQC (APQC, 2016).

O objetivo deste trabalho é elaborar um modelo de referência que contemplem os processos de operação de um PSS a partir de um modelo de referência de processos, o Process Classification Framework da APQC (APQC, 2016).

O trabalho está estruturado como se segue. A Seção 2 apresenta a revisão bibliográfica relativa aos temas de servitização, modelos de processos de negócio, modelos de desenvolvimento de PSS. A metodologia é descrita na seção 3. Os resultados e a discussão são apresentados na seção 4 . Finalmente, a seção 5 expõe as considerações finais e os trabalhos futuros a serem desenvolvidos.

\section{REVISÃO BIBLIOGRÁFICA}

O fornecimento de PSS requer mudança no modelo de negócio para a venda de produtos puros para o oferecimento de soluções integradas de produtos e serviços (BOEHM; THOMAS, 2013, p.252) . Essa mudança no paradigma da oferta é definida por (Baines et al., (2009) como servitização. Empresas que querem aumentar o valor oferecido aos clientes podem optar, então, pela servitização, visto que é uma oportunidade de atender as necessidades dos clientes e stakeholders por meio do valor percebido no uso.

Um dos maiores desafios na servitização é que a nova definição do modelo de negócios seja capaz de atender aos processos de todo o ciclo de vida do PSS, incluindo os de operação e fim de vida (PIERONI, 2017). As fases do ciclo de vida do PSS são: beginning of life (BOL) como desenvolvimento, produção e implementação do PSS, vendas; middle of life (MOL) com a venda, uso e manutenção dos serviços do PSS; e $o$ end of life (EOL) - que inclui o reuso e a disposição do PSS (PEZZOTTA; CAVALIERI; GAIARDELLI, 2012, p.222)

Não são encontrados na literatura modelos genéricos o suficiente para servir como referência a toda empresa que deseja definir seu modelo de processo no desenvolvimento de PSS, visto que os modelos possuem diferentes orientações (MARQUES et al., 2016, p. 146). Além disso, poucos deles abordam as atividades das fases de MOL e EOL. Por exemplo, Alonso-Rasgado e Thompson, (2006) propõem um modelo para o desenvolvimento de PSS sob encomenda; o 
modelo de Sakao e Shimomura, (2007) tem orientação voltada ao desenvolvimento de serviços; Brezet et al., (2001) apresentam um modelo que enfatiza a perspectiva ambiental e, por isso, apresenta algumas atividades de operações de PSS como a avaliação do processo de desenvolvimento, análise do impacto ambiental e observação das respostas do mercado com o PSS em operação; Becker, Beverungen e Knackstedt, (2010) apresentam um modelo para PSS aplicado à combinação entre uma empresa de manufatura de caminhão e uma consultoria logística, em que além de ser específico à um negócio, não apresenta apoio entre a solução integrada e o desenvolvimento e não apresenta a definição de indicadores de desempenho.

Modelos de referências de processos de negócio são representações da realidade que auxiliam as empresas no desenho dos seus negócios Becker, Beverungen e Knackstedt, (2010). Castagnari, (2016) avaliou quatorze modelos de referência de processos de negócio para aplicação em PSS. Tal autor sugeriu o Process Classification Framework (PCF) da APQC (APQC, 2016) como melhor opção para adaptação de modelo aos processos de operação de um PSS. O modelo de referência de processos (PFC) da APQC possui orientação para manufatura e serviços. Seus processos são organizados hierarquicamente em cinco níveis (grupo de processos, processos, subprocessos, atividades e tarefas).

O modelo possui algumas limitações, por exemplo na medição de desempenho, sendo encontrados apenas a definição de indicadores para algum dos processos, sub processos e atividades. Possui determinação dos requisitos dos clientes, atividade dedicada ao gerenciamento de relação com clientes durante o desenvolvimento e produtos e serviços apoiando a co-criação de valor. O PCF apresenta atividades relacionadas com parceiros dentro dos processos de entrega de produtos, prestação de serviços e serviço ao consumidor, apoiando a criação conjunta de valor. Um fator limitante é que o modelo prevê produtos e serviços como entidades separadas, o que limita os processos de melhoria contínua de design e entrega. Oferece apoio ao gerenciamento de conhecimento, assim como suporte limitado ao gerenciamento do ciclo de vida de soluções integradas de produtos e serviços e à viabilização de receitas por apresenta atividades dedicadas a estratégia de precificação e de estratégia de marketing (CASTAGNARI, 2016). Ainda que tenha sido escolhido como melhor modelo para aplicação em PSS, as limitações do modelo apontadas, precisarão ser revisadas e melhoradas.

\section{METODOLOGIA}

A metodologia adotada neste trabalho compreendeu a revisão bibliográfica da literatura no contexto de servitização, modelos de referência de processos de negócio e modelos de design de PSS. Para a revisão da literatura, são considerados os trabalhos provenientes das bases de dados eletrônicas Scopus e ISI/Web of Science. A escolha por essas bases de dados foi fundamentada em Falagas et al. (2008), que afirmou que elas se complementam e promovem uma adequada cobertura de trabalhos no domínio da ciência do gerenciamento.

Buscas iterativas foram conduzidas por meio da utilização de palavras-chave. Foram considerados os trabalhos de maior relevância de acordo com a própria ordenação das bases de dados. Inicialmente, foi realizada a leitura do título, do resumo (abstract) e das palavraschave. Se a proposta da pesquisa se apresentou pertinente, realizou-se então a leitura da introdução e das conclusões. Se relevantes, os trabalhos foram selecionados e incluídos na revisão da literatura, em que o trabalho foi lido por completo. Adicionalmente, pesquisas secundárias foram conduzidas para a identificação de estudos adicionais por meio do rastreamento das referências primárias. 
Ressalta-se que durante a busca pelo tópico de pesquisa de modelos de referência de processos de negócio, foram identificadas outras referências importantes concentradas em publicações encontradas em livros de Associações Industriais.

Além disso, contempla a análise de similaridade do PCF com as atividades de modelos de desenvolvimento de PSS. As atividades estão sendo consolidadas em uma pesquisa de doutorado em andamento de um dos integrantes do grupo de pesquisa, e possui o objetivo de auxiliar na proposição de um framework para criação de modelos de processos específicos de desenvolvimento de PSS. Também inclui a adição de processos/subprocessos/atividades de uma arquitetura de processos de negócio de PSS desenvolvida por Pieroni, (2017).

As atividades dos modelos de PSS foram comparadas por meio de suas descrições com as descrições das atividades do modelo de referência. Essa comparação foi realizada observando-se a presença de palavras similares, sinônimos ou se as descrições conduzem ao mesmo resultado. A Tabela 1 ilustra um exemplo de atividades similares:

Tabela 1: Exemplo de comparação entre as descrições das atividades

\begin{tabular}{|c|c|c|c|}
\hline Uు & 3.1 .1 .4 & $\begin{array}{l}\text { Análise de organizações } \\
\text { competidoras, produtos e serviços } \\
\text { substitutos/ competidores }\end{array}$ & $\begin{array}{l}\text { Examina os pontos fortes e fracos das organizações concorrentes. } \\
\text { Avalie organizações concorrentes para ofertas, estratégia de produto, } \\
\text { canais de marketing e entrega, etc. Analise a experiência de usabilidade, } \\
\text { durabilidade, USP e outros atributos-chave de produtos concorrentes e } \\
\text { substituídos. Reúna inteligência competitiva e considere alistar serviços } \\
\text { profissionais. }\end{array}$ \\
\hline \multirow{2}{*}{$\begin{array}{l}n \\
0 \\
0 \\
0 \\
0 \\
0 \\
0 \\
0 \\
0\end{array}$} & & Realizar análise de competidores & $\begin{array}{l}\text { Conduzem-se análises dos competidores do PSS em desenvolvimento } \\
\text { no projeto. }\end{array}$ \\
\hline & & $\begin{array}{l}\text { Buscar e analisar outras soluções } \\
\text { [fora do processo de } \\
\text { desenvolvimento] }\end{array}$ & $\begin{array}{l}\text { Busca-se por outras soluções disponíveis no mercado (produtos) para } \\
\text { servir como inspiração para geração de ideias de PSS. }\end{array}$ \\
\hline
\end{tabular}

A primeira atividade (do modelo de referência da tabela 1) examina outras organizações em diferentes aspectos e experiências de usabilidade e durabilidade de outros produtos, enquanto as outras duas (atividades dos modelos de PSS), tratam da análise dos competidores do PSS e da busca por outros produtos existentes no mercado. Considerou-se então que elas possuem o mesmo objetivo e, portanto, são similares.

Em seguida foram analisados os processos/subprocessos/atividades da arquitetura de processos de negócio de PSS (PIERONI, 2017) para adicioná-las ao modelo.

Dessa forma, a categoria "Comercializar produtos e serviços" foi analisada e as suas atividades foram comparadas. A categoria "Fornecer serviços" foi analisada também, mas nesse caso, foi completamente substituída pela categoria "Fornecer PSS" (PIERONI, 2017) com todo o seu conteúdo.

\section{RESULTADOS}

As categorias do modelo foram reescritas para atender às necessidades do PSS. Essas categorias são apresentadas na Tabela 2. Na primeira coluna, "Categorias PCF" são apresentadas as categorias do modelo original, enquanto na coluna "Categorias orientadas ao PSS" estão na segunda coluna. As categorias que sofreram alterações de adição de PSS, foram as de "Desenvolver produtos e serviços" e "Comercializar e Vender Produtos, Serviços" que passaram a ser "Desenvolver produtos, serviços e PSS" e "Comercializar e Vender Produtos, Serviços e PSS". A categoria de "Entregar Serviços" passou a ser "Fornecer PSS". Dessas, 
apenas Comercializar e Vender Produtos e Serviços" e "Fornecer PSS" foram analisadas, pois o foco do trabalho é nas atividades de MOL e EOL.

Tabela 2: Alinhamento entre as categorias do modelo de referência de processos (PCF) e as categorias orientadas ao PSS

\begin{tabular}{|l|l|l|}
\hline ID & Categorias PCF (Versão 7.05) & Categorias orientadas ao PSS \\
\hline 1.0 & Desenvolver Visão e Estratégia & Desenvolver Visão e Estratégia \\
\hline 2.0 & Desenvolver e Gerenciar Produtos e Serviços & Desenvolver e Gerenciar Produtos, Serviços e PSS \\
\hline 3.0 & Comercializar e Vender Produtos E Serviços & Comercializar e Vender Produtos, Serviços e PSS \\
\hline 4.0 & Entregar Produtos Físicos & Entregar Produtos Físicos \\
\hline 5.0 & Entregar Serviços & Fornecer PSS \\
\hline 6.0 & Gerenciar Atendimento ao Cliente & Gerenciar Atendimento ao Cliente \\
\hline 7.0 & Desenvolver e Gerenciar Capital Humano & Desenvolver e Gerenciar Capital Humano \\
\hline 8.0 & Gerenciar Tecnologia da Informação & Gerenciar Tecnologia da Informação \\
\hline 9.0 & Gerenciar Recursos Financeiros & Gerenciar Recursos Financeiros \\
\hline 10.0 & Adquirir, Construir e Gerenciar Ativos & Adquirir, Construir e Gerenciar Ativos \\
\hline 11.0 & Gerenciar Risco Empresarial, Conformidade, Remediação & Gerenciar Risco Empresarial, Conformidade, Remediação \\
e Resiliência & Resiliência \\
\hline 12.0 & Gerenciar Relações Externas & Gerenciar Relações Externas \\
\hline 13.0 & Desenvolver e Gerenciar as Capacidades Empresariais & Desenvolver e Gerenciar as Capacidades Empresariais \\
\hline
\end{tabular}

O resultado da análise da categoria 3.0 - "Comercializar e Vender Produtos, Serviços e PSS" é mostrado na Tabela 3 primeira coluna da tabela estão os processos do modelo de referência que são similares às atividades comparadas, que se encontram na segunda coluna. Os processos/ subprocessos e as atividades que apresentam similaridade foram indicados na tabela. Por exemplo "Realizar análise de inteligência de clientes e de mercado" e "realizar análise de mercado". Também foram adicionados processos/subprocessos e atividades à categoria. Destacados em cinza escuro estão as atividades de modelos de desenvolvimento de PSS e em cinza claro as atividades desenvolvidas na arquitetura de processos de negócio.

Tabela 3. Processos/ subprocessos e atividades da Categoria de Comercializar produtos Serviços e PSS comparados aos similares no modelo de referência (continua)

\begin{tabular}{|l|l|l|}
\hline 3.0 & \multicolumn{1}{|c|}{ Comercializar Produtos e Serviços } & \multicolumn{1}{c|}{ Comercializar Produtos, serviços e PSS } \\
\hline 3.1 & Entender mercados, consumidores e capabilidades & Entender mercados, consumidores e capabilidades \\
\hline 3.1 .1 & Realizar análise de inteligência de clientes e de mercado & Realizar análise de mercado \\
\hline 3.1 .1 .1 & Conduzir pesquisas de mercado & Conduzir pesquisas de mercado \\
\hline 3.1 .1 .1 .1 & & Identificar, analisar e decidir necessidades \\
\hline 3.1 .1 .1 .2 & & $\begin{array}{l}\text { Identificar, analisar e classificar pessoas externas } \\
\text { relevantes ao processo }\end{array}$ \\
\hline 3.1.1.4 & $\begin{array}{l}\text { Analisar organizações competidoras, produtos/ serviços } \\
\text { competidores/ substitutos }\end{array}$ & Realizar análise de competidores \\
\hline 3.1 .1 .4 .1 & & $\begin{array}{l}\text { Buscar e analisar outras soluções [fora do processo de } \\
\text { desenvolvimento] }\end{array}$ \\
\hline 3.1 .1 .5 & Avaliar produtos/serviços existentes & Avaliar produtos/serviços existentes \\
\hline 3.1 .1 .5 .1 & & Analisar serviços existentes \\
\hline 3.1 .1 .6 & Avaliar o ambiente de negócios interno e externo & Analisar o ambiente das pessoas \\
\hline 3.1 .2 & Avaliar e priorizar oportunidades de mercado & Avaliar e priorizar oportunidades de mercado \\
\hline 3.1 .2 .2 & Determinar segmentos-alvo & Dividir o mercado \\
\hline 3.1 .2 .2 .1 & & Analisar o contexto \\
\hline 3.1 .3 & & Criar Persona \\
\hline 3.2 & Desenvolver estratégia de marketing & Desenvolver estratégia de marketing \\
\hline 3.2 .1 & Definir oferta e proposta de valor do cliente & Avaliar o valor \\
\hline 3.3 & Desenvolver e planejar plano de vendas & Planejar estratégia \\
\hline & & \\
\hline
\end{tabular}


Tabela 3. (Continuação) Processos/ subprocessos e atividades da Categoria de Comercializar produtos Serviços e PSS comparados aos similares no modelo de referência

\begin{tabular}{|c|c|c|}
\hline 3.5 & Desenvolver e gerenciar plano de vendas & Desenvolver e gerenciar plano de vendas \\
\hline 3.5 .1 & Gerenciar oportunidades & Gerenciar oportunidades \\
\hline 3.5.1.1 & Identificar clientes potenciais & Identificar clientes potenciais \\
\hline 3.5.1.2 & Identifiar/receber oportunidades & Identificar oportunidades de vendas de produto \\
\hline 3.5.1.3 & & Identificar oportunidades de vendas de PSS \\
\hline 3.5 .2 & Gerenciar cliente e contas & Gerenciar carteiras de clientes \\
\hline 3.5.2.2 & Desenvolver plano de vendas/contas chave & Desenvolver planos de vendas por clientes \\
\hline 3.5 .2 .4 & Gerenciar relacionamentro com clientes & Gerenciar relacionamento com clientes \\
\hline 3.5.2.5 & Gerenciar dados mestre dos clientes & Gerenciar dados dos clientes \\
\hline 3.5 .3 & $\begin{array}{l}\text { Desenvolver e gerenciar propostas de vendas, lances e } \\
\text { orçamentos }\end{array}$ & Gerenciar vendas aos clientes \\
\hline 3.5.3.1 & & Realizar visita ao cliente e proposta de venda \\
\hline 3.5.3.2 & & Avaliar a necessidade de adequação do ambiente \\
\hline 3.5.3.3 & & Efetuar a transação comercial do PSS \\
\hline 3.5.3.4 & & Elaborar contrato \\
\hline 3.5.3.5 & & Fornecer visão das funções \\
\hline 3.5 .3 .5 & & Assinar contrato \\
\hline 3.5.3.6 & & Registrar resultado do processo de vendas \\
\hline 3.5.3.7 & & Informar pessoas externas relevantes ao processo \\
\hline 3.5.3.10 & Desenvolver estimativas de preços e agendamento & Definir preço dos novos produtos e serviços \\
\hline 3.5 .3 .16 & & Analisar sensibilidade de preço do PSS \\
\hline 3.5 .4 & Gerenciar pedidos de vendas & Gerenciar pedidos de vendas \\
\hline 3.5 .4 .1 & & Gerenciar informações dos clientes \\
\hline 3.5 .4 .2 & & Avaliar disponibilidade do ativo de PSS e reservar \\
\hline 3.5.4.2.1 & & Gerenciar contratos cadastrados para renovações \\
\hline 3.5 .5 & Gerenciar parcerias/ alianças & Gerenciar parcerias de vendas \\
\hline 3.5.5.1 & & Elaborar previsão de vendas por parceiros \\
\hline 3.5.5.3 & Avaliar resultado das parcerias/alianças & Avaliar resultados da parceria \\
\hline 3.5.5.4 & Gerenciar dados-mestres de parcerias/alianças & Gerenciar dados de parceiros \\
\hline 3.5 .6 & & Gerenciar força de vendas \\
\hline 3.5.6.1 & & Definir alocação de recursos de vendas \\
\hline 3.5.6.2 & & Estabelecer plano de remuneração da força de vendas \\
\hline
\end{tabular}

O resultado da modificação na categoria 5.0 - "Fornecer PSS": A Tabela 4 apresenta a categoria 5 completamente modificada. Essas categorias contêm processos e atividades relacionadas ao planejamento de recursos para o fornecimento de PSS, treinamento de funcionários, manutenção dos serviços e equipamento, entrega e instalação do PSS, alteração do ambiente de operação de PSS entre outros que podem ser vistos na tabela. Os processos foram detalhados em subprocessos e atividades, entretanto a tabela 3 ilustra apenas até o nível de subprocessos.

Tabela 4. Processos da categoria "Fornecer PSS", incorporada ao PCF (continua)

\subsection{Fornecer PSS}

5.1 Planejar recursos para fornecimento de PSS

5.1.1 Gerenciar demanda de recursos para novos PSS

5.1.2 Criar plano de recursos

5.1.3 Disponibilizar recursos humanos para fornecimento de PSS

5.1.4 Gerenciar ativos (equipamentos) para PSS

5.1.5 Gerenciar peças de reposição

5.1.6 Gerenciar parcerias 
Tabela 4. (Continuação) Processos da categoria "Fornecer PSS", incorporada ao PCF

5.2 Ativar contrato de PSS

5.2.1 Programar recursos para ativação

5.2.2 Realizar ativação de contrato específico

5.2.3 Gerenciar as ativações de contratos

5.3 Garantir disponibilidade de uso

5.3.1 Programar e executar manutenção preventiva

5.3.2 Programar e executar manutenção corretiva

5.3.3 Programar e executar atualizações tecnológicas

5.3.4 Gerenciar manutenções e atualizações

5.4 Finalizar contrato de PSS

5.4.1 Finalizar contrato e retirar equipamento do cliente

5.4.2 Recondicionar equipamento retornado

5.5 Fornecer experiência de uso

5.5.1 Planejar e gerenciar equipe de experiência de uso

5.5.2 Gerenciar solicitações do cliente

5.5.3 Medir satisfação do cliente quanto às reclamações

5.5.4 Medir satisfação do cliente quanto ao PSS

5.5.5 Planejar e fornecer atendimento presencial ao cliente

5.6 Fornecer serviços adicionais

5.6.1 Fornecer treinamento adicional

5.6.2 Fornecer laudo remoto

\section{COMENTÁRIOS FINAIS}

Foram avaliadas as atividades referentes às duas principais categorias de processos de middle of life de um PSS. Pode-se observar um grande potencial do modelo de referência em relação às atividades de comercialização e venda dos produtos e serviços e PSS, principalmente por já conter o direcionamento à oferta de serviços além da manufatura. $\mathrm{O}$ modelo não apresenta algumas atividades referentes às responsabilidades do fornecedor (elaboração de contrato, manutenção dos serviços, treinamento dos funcionários), que são de extrema importância para a operação de um PSS, havendo a necessidade de adição.

Apesar de considerar os serviços, o modelo considera apenas os serviços de apoio ao produto após a venda, diferente do PSS, em que o serviço faz parte da oferta de valor. Havendo assim a necessidade de alteração da descrição de algumas dessas atividades referentes aos serviços

Existe grande potencial para que as atividades de desenvolvimento de PSS sejam incorporadas ao modelo, bem como a avaliação das outras categorias, para que atendam às necessidades dos processos de negócio de um PSS, não apenas os de operação.

Para trabalhos futuros serão avaliadas de desenvolvimento dos modelos de PSS, para incorporação no modelo e as demais categorias. Também serão avaliados os indicadores de desempenho e os papéis do pessoal dentro da organização em todo o contexto de servitização. 


\section{REFERÊNCIAS}

ALONSO-RASGADO, T.; THOMPSON, G. A rapid design process for Total Care Product creation. Journal of Engineering Design, v. 17, n. 6, p. 509-531, 2006.

APQC. CROSS INDUSTRY PROCESS CLASSIFICATION FRAMEWORK® Version 7.0.2 OVERVIEW. n. April, 2016.

BAINES, T. et al. The servitization of manufacturing: A review of literature and reflection on future challenges. Journal of Manufacturing Technology Management, v. 20, n. 5, p. 547$567,2009$.

BAINES, T. S. et al. State-of-the-art in product-service systems. Proceedings of the Institution of Mechanical Engineers, Part B: Journal of Engineering Manufacture, v. 221, n. 10, p. 1543-1552, 2007.

BECKER, J.; BEVERUNGEN, D. F.; KNACKSTEDT, R. The challenge of conceptual modeling for product-service systems: Status-quo and perspectives for reference models and modeling languages. Information Systems and e-Business Management, v. 8, n. 1, p. 33-66, 2010 .

BOEHM, M.; THOMAS, O. Looking beyond the rim of one's teacup: A multidisciplinary literature review of Product-Service Systems in Information Systems, Business Management, and Engineering \& DesignJournal of Cleaner Production, 2013.

BREZET, J. C. et al. The Design of Eco-Efficient Services. [s.l: s.n.].

CASTAGNARI, P. V. A. Análise de modelos de referência de processos de negócio para apoiar a arquitetura de processos de Sistemas Produto-Serviço (PSS) e a implantação de sensores viabilizadores das tecnologias de Internet of Things (IoT). [s.l.] University of São Paulo, 2016.

FALAGAS, M. E. et al. Comparison of PubMed, Scopus, Web of Science, and Google Scholar: strengths and weaknesses. The FASEB Journal, v. 22, n. 2, p. 338-342, 2008.

MARQUES, C. A. N. et al. Comparing PSS Design Models Based on Content Analysis. Procedia CIRP. Anais...2016

PEZZOTTA, G.; CAVALIERI, S.; GAIARDELLI, P. A spiral process model to engineer a product service system: An explorative analysis through case studies. CIRP Journal of Manufacturing Science and Technology, v. 5, n. 3, p. 214-225, 2012.

PIERONI, M. et al. Transforming a Traditional Product Offer into PSS: A Practical Application. Procedia CIRP. Anais...2016

PIERONI, M. Proposal of a Business Process Architecture (BPA) Development Method for supporting the transition of manufacturing companies into Product-Service System (PSS) providers. [s.1.] University of São Paulo, 2017.

SAKAO, T.; SHIMOMURA, Y. Service Engineering: a novel engineering discipline for producers to increase value combining service and product. Journal of Cleaner Production, v. 15, n. 6, p. 590-604, 2007. 


\section{AGRADECIMENTOS}

Este projeto é apoiado pelo processo no 2015/23094-6, Fundação de Amparo à Pesquisa do Estado de São Paulo (FAPESP). Os autores agradecem o apoio da FAPESP e da Coordenação de Aperfeiçoamento de Pessoal de Nível Superior (CAPES). 